\title{
Apropiación tecnológica de los profesores: el uso de recursos educativos abiertos
}

Marisol Guacaneme-Mahecha

Colegio Bilingüe Richmond, Colombia marisolguacaneme@gmail.com

Marcela Georgina Gómez-Zermeño

Tecnológico de Monterrey, México marcela.gomez@itesm.mx

\section{David Zambrano-Izquierdo}

Tecnológico de Monterrey, México zambranodavid.izquierdo@gmail.com

\section{Resumen}

El objetivo de la investigación es analizar el proceso de apropiación tecnológica de un grupo de profesores de escuela media, de un colegio bilingüe en Bogotá (Colombia), en relación con la selección, integración y aplicación de recursos abiertos. La pregunta de investigación que guió el estudio fue: ¿cómo ocurre la apropiación tecnológica en los profesores que incorporan recursos educativos abiertos en la educación básica en un colegio bilingüe de la ciudad de Bogotá? Las técnicas de recolección de datos fueron la entrevista semiestructurada y la observación no participativa, las cuales fueron aplicadas a cuatro docentes y veinte estudiantes de la escuela secundaria. Se analizaron tres ejes de investigación: apropiación tecnológica, ambientes de aprendizaje y recursos educativos abiertos. Los hallazgos encontrados arrojaron que los docentes se encuentran familiarizados con las herramientas tecnológicas, hacen un buen uso de ellas, manejan computadores y proyectores dentro de su salón, y hacen inclusión permanente de recursos de apoyo a sus clases.

\section{Palabras clave}

Investigación escolar; investigación de aula; TIC en educación; educación básica; recursos educativos abiertos (Fuente: Tesauro de la Unesco).

Recepción: 12-03-2015 | Envío a pares: 13-10-2015 | Aceptación: 15-12-2015 | Aprobación: 18-12-2015 


\title{
Technological Appropriation by Teachers: The Use of Open Educational Resources
}

\begin{abstract}
The objective of this study was to analyze the process of technological appropriation on the part of a group of middle school teachers at a bilingual school in Bogota (Colombia), specifically in terms of their selection, addition and implementation of open resources. The research question guiding the study was: How does technological appropriation occur among teachers who incorporate open educational resources into basic education at a bilingual school in Bogota? Semi-structured interviews and non-participant observation were the data collection techniques used. They were applied to four high school teachers and twenty students. Three areas of research were analyzed: technological appropriation, learning environments and open educational resources. The findings show the teachers are familiar with technological tools, make good use of them, operate computers and projectors in their classrooms, and constantly include resources to support their class work.
\end{abstract}

\section{Keywords}

School research; classroom research; ICT in education; basic education; open educational resources (Source: Unesco Thesaurus). 


\section{Apropriação tecnológica dos professores: o uso de recursos educativos abertos}

\section{Resumo}

O objetivo desta pesquisa é analisar o processo de apropriação tecnológica de um grupo de professores de escola média, de um colégio bilíngue em Bogotá (Colômbia), com relação a seleção, integração e aplicação de recursos abertos. A pergunta de pesquisa que orientou o estudo foi: como a apropriação tecnológica ocorre nos professores que incorporam recursos educativos abertos na educação básica num colégio bilíngue da cidade de Bogotá? As técnicas de coleta de dados foram a entrevista semiestruturada e a observação não participativa, as quais foram aplicadas a quatro docentes e vinte estudantes da escola secundária. Analisaram-se três eixos de pesquisa: apropriação tecnológica, ambientes de aprendizagem e recursos educativos abertos. Os achados demonstraram que os docentes se encontram familiarizados com as ferramentas tecnológicas, fazem um bom uso delas, trabalham com computadorese projetores dentro da sala de aula, e fazem inclusão permanente de recursos de apoio em suas aulas.

\section{Palavras-chave}

Pesquisa escolar; pesquisa de sala de aula; TIC em educação; educação básica; recursos educativos abertos (Fonte: Tesauro da Unesco). 


\section{Introducción}

En los últimos años se han suscitado transformaciones significativas en los centros educativos de Latinoamérica, las cuales representan cambios de posturas en el proceso de enseñanza-aprendizaje, tanto en docentes como en alumnos. El utilizar herramientas tecnológicas y recursos educativos abiertos (REA) contribuye al mejoramiento de los procesos educativos, y los estudiantes se convierten en alumnos activos y partícipes de su proceso de aprendizaje (Gómez-Zermeño, 2012).

En Colombia, la Asamblea General por la Educación del año 2007 recopiló los aportes de más de 20,000 colombianos representantes de todos los sectores de la sociedad al Plan Nacional Decenal de Educación 2006-2015 (Ministerio de Educación Nacional, 2008). El encuentro mencionado se llevó a cabo para atender la preocupación por parte del sector educativo colombiano sobre los procesos que se estaban propiciando; se veía necesario que el país estuviera a la vanguardia de los nuevos procesos de enseñanza-aprendizaje, los cuales eran mediados por tecnologías. Lo mencionado hizo surgir la inquietud por explorar, en un colegio bilingüe de Bogotá, cómo ocurría la apropiación tecnológica y cómo los docentes hacían uso de los recursos educativos abiertos.

Según el Ministerio de Comunicaciones (Barón y Gómez, 2012), no es suficiente contar con una excelente infraestructura o acceso si no se trabaja hacia la mejora en la calidad de vida con relación al cierre de brechas y uso masivo de la tecnología. Esta investigación se enfocó en indagar los conocimientos que posee un grupo de profesores sobre la apropiación tecnológica y los recursos educativos abiertos, con el objetivo de identificar si estos estaban siendo aplicados dentro de su práctica docente y en el currículo. Los resultados de la investigación son útiles para conocer las deficiencias y necesidades de los docentes de una institución educativa; además, permitirán establecer capacitaciones al interior de la institución sobre ese aspecto.
De acuerdo con lo mencionado, se generó la pregunta de investigación: ¿cómo ocurre la apropiación tecnológica en los profesores que incorporan recursos educativos abiertos en la educación básica en un colegio bilingüe de la ciudad de Bogotá?

A través del proceso de investigación, se buscó analizar los factores que deben considerar los profesores al integrar los REA en los ambientes de aprendizaje con tecnología; asimismo, se buscó identificar las dificultades que enfrentan los docentes de educación secundaria al integrar los REA en sus cursos. Se planteó como objetivo general analizar el proceso de apropiación tecnológica de cuatro profesores de escuela media, de un colegio bilingüe en Bogotá (Colombia), en relación con la selección, integración y aplicación de recursos abiertos.

\section{Marco teórico}

\section{Apropiación tecnológica}

Actualmente, la tecnología forma parte indispensable de la adquisición del conocimiento, la cual se ha convertido en una práctica social ya sea entre pares o en una comunidad de aprendizaje; esta tecnología debe ser usada e incorporada por cada uno de los integrantes de la comunidad, como lo mencionan Colás y Jiménez (2008, citado por Celaya, Lozano y Ramírez, 2010), la apropiación es definida como tomar algo que pertenece a otro y hacerlo suyo. Al apropiarse de la herramienta, el individuo hace que el conocimiento adquirido tome relevancia. El aprendizaje se muestra como una estructura social. En la apropiación tecnológica entran en juego factores tales como la disposición, la motivación y el interés que cada individuo le aplique. Por otro lado, es importante conocer y entender el fenómeno de la apropiación tecnológica desde tres fases descritas por Lizama (2010): exploración, juego y apropiación.

Dentro de la apropiación tecnológica, cabe mencionar que la comunidad atraviesa fases, ya que el profesorado o el personal a cargo deben convertirse 
en miembros activos de este cambio educativo. Para que esta apropiación tenga significado, la comunidad debe pasar por las siguientes fases, según Hooper y Rieber (1995): familiarización, utilización, integración, reorientación y evaluación.

Debido a los cambios que han generado nuevas formas de enseñanza, se han abierto nuevos espacios de socialización pedagógica, estos nuevos espacios son llamados ambientes de aprendizaje, los cuales son el entorno en el cual se desarrolla el individuo, este puede ser su familia, comunidad y su entorno educativo. Según Duarte (2003), el ambiente se genera a través de la interacción del hombre con el entorno, donde se involucra la acción pedagógica; bajo estas condiciones, el sujeto está en la capacidad de reflexionar sobre su acción y así mismo sobre la de los demás en relación con el ambiente.

Un ambiente de aprendizaje es el conjunto de elementos materiales, tales como: la arquitectura, el equipamiento y el lugar; los elementos culturales; los elementos sociales, que permiten la interactividad, la comunicación y el trabajo en equipo; los elementos de tiempo, que incluyen la planeación y el momento en el que se lleva a cabo el aprendizaje y, finalmente, el contenido académico, que también es conocido como plan de estudios (Woolfolk, 2006, citado por Ramírez, 2012).

Los ambientes de aprendizaje cuentan con componentes que les dan características propias; según Ramírez (2012), los componentes de los ambientes de aprendizaje son: lugares, roles diferenciados, equipo de apoyo, integración creativa, pensar diferente en cuanto a evaluación y comunicación, tener un sentido de trascendencia y mirada de Impacto; es brindar todas las herramientas necesarias para el crecimiento personal, social y laboral.

Es importante mencionar que los componentes de los ambientes de aprendizaje no se limitan a un espacio físico; según Briceño (2009), hacen parte de estos ambientes: las relaciones comunicacionales humanas e interpersonales, el intercambio de ideas y los fenómenos educativos; además, entran en juego los objetos y las circunstancias, que son factores que rodean al sujeto y a su comunidad para desarrollar conocimiento, habilidades y destrezas.

\section{Recursos educativos abiertos}

Los recursos educativos abiertos, también llamados open educational resources (OER), pueden ser usados por todas las personas alrededor del mundo, su funcionabilidad y acceso han hecho que tomen fuerza dentro del ámbito educativo, estos son gratuitos y se encuentran en Internet. Las transformaciones sociales presentes en la sociedad moderna hacen que los REA tomen gran impacto dentro de la misma. Según Mortera (2011), los REA representan una tendencia nueva y con gran empuje en la sociedad del conocimiento, la cual se produce dentro del ámbito educativo y tiene un gran impacto dentro de los procesos de aprendizaje en las diferentes modalidades.

Como lo mencionan Rivera, López y Ramírez (2011), los REA surgieron a principios de la pasada década y su objetivo radicaba en compartir información para disminuir el espacio entre aquellas comunidades que no tenían un acceso a información de calidad y comunidades con acceso a esta.

Es esencial entender el término abierto como una característica de la información que avala el acceso en tres niveles: existe libertad en su uso, sin importar el propósito que se le dé; libre acceso, manipulación y modificación del recurso; y libre distribución de la información a la cual se accedió o como una versión modificada de la misma (Ramírez y Burgos, 2012).

\section{Metodología}

Para la investigación, se consideró el método cualitativo ya que, según Mayan (2001), indaga sobre las experiencias de los individuos en su contexto real, sin manipular el entorno de estudio. Se realizó un estudio de caso, el cual radica en hacer un análisis y descripción a profundidad de un sistema 
delimitado, que se caracteriza por su riqueza en la descripción (Valenzuela y Flores, 2012). El estudio de casos se relaciona con uno o más casos de la vida real (Yin, 2006). En esta investigación se llevó a cabo un estudio de caso descriptivo, que busca identificar y describir los distintos factores que ejercen influencia en el fenómeno estudiado.

El estudio de casos se basó en analizar los fenómenos de un hecho real y específico, en este caso, la unidad de análisis corresponde a un colegio bilingüe de la ciudad de Bogotá, sobre cómo ocurre la apropiación tecnológica y cómo se utilizan los REA dentro del ámbito escolar.

La población estuvo conformada por los docentes y alumnos de un colegio bilingüe en la ciudad de Bogotá (Colombia). Según Stake (1994, citado por Yin, 2006), la selección de la fuentes es un paso crítico para llevar a cabo el estudio de casos. Los informantes fueron cuatro docentes de las áreas de Matemáticas, Inglés, Español y Ciencias, quienes impartían clases en los grados cuarto a séptimo de la escuela media; así mismo, participaron veinte estudiantes de la escuela, cuyas aportaciones sirvieron para trabajar desde otro punto de vista.

Se utilizó la entrevista semiestructurada y la observación no participativa como técnicas de recolección de datos.

La entrevista estuvo enfocada a recabar información de tres aspectos: apropiación tecnológica, ambientes de aprendizaje y uso de recursos educativos abiertos. Para el análisis de la apropiación tecnológica se adaptaron los niveles propuestos por Hooper y Rieber (1995): familiarización, utilización, integración y reorientación. Para el análisis de diseño de ambientes de aprendizaje con tecnologías, se consideraron los siguientes indicadores: concepto, componentes de los diseños de ambientes de aprendizaje, integración de la tecnología en ambientes de aprendizaje. Para la categoría de recursos educativos abiertos se utilizaron los siguientes niveles: concep- to y características. Se realizó una prueba piloto con dos docentes de la institución diferentes a la muestra, los resultados permitieron validar la estructura de la entrevista.

Por otro lado, la observación no participativa o directa fue realizada a los cuatro docentes y a los estudiantes en las clases donde se planeó la utilización de los recursos educativos abiertos. Se pretendía observar, además, cómo eran dadas las instrucciones, qué tan pertinente era el contenido y cómo era la postura del estudiante frente a estas prácticas.

Los items que se usaron para la observación fueron: información general de los participantes (nombre, sexo, edad, asignatura que enseña, nivel educativo, si habla otro idioma, años de experiencia, años en el colegio); descripción del aula (infraestructura, herramientas tecnológicas); manejo por parte del docente de las herramientas tecnológicas y uso de recursos educativos abiertos. Las observaciones fueron recogidas en una ficha de observación.

Finalmente, se hizo una validación de la información. Como menciona Cifuentes (2011), la validación se desarrolla en procesos de acción, interacción, participación y triangulación, a partir del contexto donde se analiza el hecho social. Por último, se realizó la triangulación metodológica dentro de métodos, como lo mencionan Valenzuela y Flores (2012), en el nivel de diseño: a partir de esta se identifica cuál fue la apreciación dada por cada participante sobre cada pregunta y se contrasta con las afirmaciones de los teóricos, la cual favorece la confiabilidad de los resultados y permite contrastarlos con la teoría.

Para el análisis de la fiabilidad y la validez de los instrumentos, se hizo uso de múltiples fuentes de evidencia, y se contó con la revisión del reporte preliminar del estudio de caso por informantes clave.

\section{Resultados}

Para conocer la manera en que ocurre la apropiación tecnológica en los profesores, se llevó a cabo 
la observación en el aula de cada docente dentro de su horario de clases. El investigador estuvo atento al desarrollo de la sesión y al uso que el docente daba a las herramientas tecnológicas así como al empleo de los REA.

En términos generales, se observó que cada docente aplicó un REA como apoyo para explicar las temáticas sujetas al plan bimestral. En el aula, el profesor informó a sus alumnos que el tema por tratar se explicaría haciendo uso de recursos educativos abiertos, y se dio una breve explicación de los mismos. El procedimiento para utilizar los REA en el aula fue como introducción al tema propuesto para la clase, y la forma de evaluarlo fue un trabajo colectivo, realizado en Google Docs acerca del tema visto. A partir de estas actividades, fue posible documentar las acciones de los participantes; las entrevistas permitieron indagar sobre la percepción de los participantes en cuando a las categorías teóricas sugeridas.

Respectó a la apropiación tecnológica, se encontró que la mayoría de los estudiantes están familiarizados con la tecnología, ya que tienen acceso directo a ella y poseen varios dispositivos; esto se debe en gran parte al apoyo que les brindan sus padres. Por otra parte, los docentes afirman que hacen un buen uso de la tecnología, utilizan recursos tales como computadores portátiles, Internet y herramientas como Wikis, Blogs, plataformas virtuales, tableros inteligentes y Video Beam. Según Laffey (2004), el marco sociocultural sugiere que el camino de la apropiación no es solo lineal, y que las herramientas no deben ser dominadas, sino apropiadas por los individuos. Se advirtió que tanto estudiantes como docentes hacen un excelente uso de la tecnología; los estudiantes observados cuentan con aparatos electrónicos de alta gama y, en algunas ocasiones, pueden sacarlos para buscar contenidos relevantes; los docentes, por su parte, en su salón de clase poseen un video proyector con salida de audio y un computador, del cual hacen un buen uso. En general, los docentes y estudiantes mostraron un adecuado y flexible uso de dichas herramientas.
Por otra parte, las fortalezas que encuentran los profesores en la utilización de herramientas tecnológicas y recursos educativos abiertos en su práctica docente son la gran variedad de información a la que se puede acceder y el conocimiento que los estudiantes tienen sobre su uso. Como debilidades se encuentran dificultades de tipo técnico y la continua actualización de la tecnología. En la observación a los docentes se notó la versatilidad de la clase y la participación activa de los estudiantes; son clases motivadoras, realizadas con agilidad; el docente maneja la clase según su criterio y la utilización de aparatos electrónicos le aligera la carga ya que intercala clase magistral con videos, canciones, ejercicios, etc. Como lo mencionan Gutiérrez y Quiroz (2007), la apropiación tecnológica se define como la interacción entre sujeto y un entorno, donde cada individuo utiliza los sistemas tecnológicos de acuerdo con sus ideales e intereses.

Adicionalmente, los maestros afirmaron que han integrado los recursos educativos abiertos en sus clases, haciendo uso de la Internet, para crear blogs, páginas web, videos; así mismo, a diario utilizan plataformas de las diferentes editoriales que están relacionadas con el tema y como consulta. Dentro de la observación realizada se percibió que a partir del tema impartido por el docente, se hacía el uso de los video-proyectores para cada actividad orientada desde cada una de las materias impartidas por los profesores tomados como muestra; así, en Inglés se trabajó una canción con verbos en pasado; en Español, una narración; en Ciencias, la estructura del átomo, y en Matemáticas, hicieron uso de la plataforma virtual. Según Hooper y Rieber (1995), la integración es la fusión del saber teórico que será dispuesto y contrastado con la utilización de herramientas tecnológicas.

Cabe señalar que los estudiantes pueden utilizar los recursos educativos abiertos para construir conocimiento, ya que a partir de estos se pueden realizar investigaciones sobre temas desconocidos y aclarar dudas; a su vez, los REA estimulan 
los conocimientos de los estudiantes y ayudan a entender mejor un tema determinado. Según Weingardt (2004, citado por Montes y Ochoa, 2006), "la apropiación de la tecnología permite mejorar y hacer más fácil la transmisión de contenidos educativos siempre y cuando el docente diseñe y disponga dichos contenidos de manera clara y eficiente" ( $p$. 89). Los docentes argumentan que pueden usar videos o hacer presentaciones como apoyo de sus clases; para ellos, los recursos educativos abiertos son una gran herramienta, la cual puede servir para la investigación y genera motivación para docentes y estudiantes, quienes se convierten en responsables de su proceso. Además, afirman que los estudiantes son los principales usuarios de la tecnología. Por otro lado, se percibió que los recursos educativos abiertos eran usados para construir conocimiento; a partir de su utilización y enfoque, los estudiantes están en la condición de adquirir información, ya que estos recursos se muestran atractivos para ellos, lo cual genera una mayor motivación a la hora de aprender. Se percibió un rol activo y participativo por parte del alumnado, son niños con muchos cuestionamientos, agentes activos de su propio aprendizaje; en repetidas oportunidades hacían sugerencias de algunas páginas y ayudaban a los maestros a buscar palabras desconocidas desde sus celulares. Según Colás, Rodríguez y Jiménez (2005), la apropiación es planteada como una herramienta cultural, que es adaptada por los individuos, y constituye la manera en la cual interpretan la realidad, llegando a ser la base de su aprendizaje.

Respecto de los ambientes de aprendizaje, se encontró que para los docentes son lugares en los cuales el estudiante se siente cómodo, lleno de estímulos y donde son protagonistas de su proceso de aprendizaje. Esto se complementa con lo que señala Briseño (2009): los ambientes de aprendizaje son escenarios que logran una transformación, en los cuales confluyen la intención de formar y de enseñar, permitiendo así que se generen cambios importantes en métodos, teorías de aprendizaje y estrategias.
Mencionan los docentes que dentro de los elementos que debe incluir un ambiente de aprendizaje se encuentran: computadores, software para este tipo de prácticas, aislamiento acústico, espacio adecuado, recursos - no solamente tecnológicos sino de otra índole, como una biblioteca en el salón de clase, diccionarios, distintos tipos de libros-; materiales como cartulinas y material didáctico manipulativo; comodidad, claridad, seguridad, todo esto para que se dé un aprendizaje significativo.

En cuando a los componentes de los ambientes de aprendizaje, los docentes afirman que deben incluir personal calificado para tal fin, una infraestructura adecuada, un tiempo pertinente, un lugar apropiado, motivación e interés tanto de los participantes como de los docentes encargados. Teóricos como Ramírez (2012) argumentan que los componentes de los ambientes de aprendizaje son: lugares, espacio, roles diferenciados, perfil del estudiante, equipo docente, equipo de apoyo, integración creativa de contenidos, estrategias de enseñanza, técnicas y recursos, contenidos, pensar diferente en cuanto a evaluación y comunicación, tener un sentido de trascendencia, visión innovadora y mirada de impacto.

Respecto a la integración de la tecnología en los ambientes de aprendizaje, los maestros argumentan que la manera de hacerlo es incorporando equipos, herramientas, plataformas y redes; la tecnología está en cada celular, en cada aparato electrónico que los niños tienen, el asunto es ponerlos al servicio de la educación, para que faciliten los procesos de aprendizaje. Atkins, Brown y Hammond (2007) afirman que los recursos educativos abiertos, como son una herramienta de gran valía para el conocimiento, son un bien público que se integra a cualquier ambiente de aprendizaje ya que ofrecen una oportunidad extraordinaria para que todos puedan usar y compartir, el conocimiento. Por otro lado, los docentes afirman que la manera en la cual pueden integrar la tecnología en un ambiente de aprendizaje con el fin de que el alumno construya conocimientos, es a partir de la planeación que 
deben realizar en cada materia, así mismo desde la enseñanza de los recursos, para que haya un dominio; el alumno debe comprender que estos recursos no solamente se utilizan para la diversión, sino para realizar investigación, debe haber las herramientas necesarias como acceso a Internet, computadores y plataformas virtuales.

Ahora bien, para los estudiantes, los REA son herramientas que proporciona la tecnología como fuentes o materiales que ayudan a la gente a buscar información, son un atajo para entender más sobre el tema con ayuda de la web, el cual puede ser utilizado tanto en el colegio como en la casa y son gratuitos, además están abiertos a todo el público.

La docente número uno, aunque hace uso de recursos educativos abiertos, no conocía su nombre; los profesores concordaron en que eran recursos gratuitos que estaban al alcance de todos a través de la red. D' Antoni (2007) se refiere a los materiales basados en la web, que se ofrecen libre y abiertamente para ser utilizados en la enseñanza, el aprendizaje y la investigación. En el proceso de implementación que se debe seguir para el uso de recursos educativos abiertos, los docentes entrevistados afirmaron que debe haber conexión a una red, el colegio debe tener una plataforma de sistemas, computadores, software, tableros inteligentes y Video Beam en los salones.

Dentro de los salones se observó que los cuatro docentes entrevistados poseen un computador y un video-proyector, todos con acceso a Internet; los estudiantes cuentan con una red de acceso exclusiva para ellos, y con celulares con conexión a la red.

En cuanto a sus características, los alumnos señalan las ventajas de usar los REA desde cualquier punto de forma gratuita, la posibilidad de buscar información de una manera rápida y aplicarla a la vida diaria; el acceso a la información es sencillo ya que todos tienen aparatos electrónicos, lo que permite generar más interés para enfocarse en la clase, buscar explicaciones de un tema que no quede claro; por otra parte, los docentes mencionan que existen muchos recursos disponibles y la motivación que brinda su uso, así como mayor participación por parte de los estudiantes. Otras características de los REA son ofrecer oportunidades y ser considerados como la llave de una transición exitosa al conocimiento basado en la economía y la sociedad en un contexto de aprendizaje (Mulder, 2007).

Dentro de las desventajas que se encontraron está no poseer un aparato electrónico o la conectividad a Internet. Para los docentes, una de las desventajas es no conocer los recursos educativos abiertos, otras desventaja que mencionan es la poca infraestructura para la utilización de recursos como lo es la conectividad a Internet y también el apego a la tecnología que pueden mostrar los estudiantes.

Según Mortera (2011), la educación moderna exige cambios significativos tanto para docentes como para estudiantes; los REA representan una tendencia nueva y con gran empuje dentro de la sociedad del conocimiento, la cual se produce dentro del ámbito educativo y tiene gran impacto dentro de los procesos de aprendizaje en las diferentes modalidades. Los REA representan un sinnúmero de funciones dentro del aula ya que son versátiles a la hora de adaptarse a cualquier contenido dentro de un currículo.

En cuanto a los usos, es decir, la aplicación que se puede dar al contexto educativo con los REA, los docentes opinan que los REA pueden ser utilizados dentro del salón de clase, ya que al conocer los temas, pueden escoger estos recursos; respecto a los recursos que utilizan, mencionan las plataformas YouTube y Gmail. Los contenidos que abordan los recursos educativos abiertos son amplios, ya que abarcan todas las áreas del conocimiento. De acuerdo con Schmidt (2007, citado por Ramírez y Burgos, 2012), usar o compartir recursos educativos abiertos no es nuevo, lo novedoso es la facilidad que brinda la tecnología para que estos recursos se puedan generar y distri- 
buir masivamente a cualquier público gracias a la Internet. En este caso, la red juega un papel importante para que los REA estén al alcance de la sociedad del conocimiento.

Para los docentes, los objetivos de los Recursos Educativos Abiertos pueden ser múltiples, como introducir un tema, exponer conceptos, dar la información general, brindar a los estudiantes las fuentes donde pueden acudir a buscar información. En cuanto a la evaluación que se puede hacer con la inclusión de recursos educativos abiertos, esta se puede llevar a cabo en el momento mismo en que se está presentando la información o partir de ella hacer una evaluación posterior; como lo mencionan Ramírez y Burgos (2011), la evaluación permite que los REA sean seleccionados y valorados en cuanto a su calidad y pertinencia.

Para concluir con este indicador, es de relevancia mencionar que los procesos educativos que se están llevando a cabo en el siglo XXI, necesariamente incluyen cualquier tipo de recurso educativo abierto. Al realizar la observación fue notorio ver a maestros empoderados de su quehacer pedagógico, personas profesionales e íntegras que han asumido el reto y los cambios que la nueva educación trae; son personas proactivas, que a partir de sus experiencias, ponen todo su conocimiento al servicio del alumno, de una manera motivante y haciendo inclusión permanente de los recursos educativos abiertos.

\section{Conclusiones}

Esta investigación tuvo por objetivo general analizar el proceso de apropiación tecnológica que realizan cuatro profesores de escuela media de un colegio bilingüe en Bogotá, desde la selección, integración y aplicación de recursos abiertos cuando son incorporados en sus clases, con el fin de aportar evidencia científica para la mejora de los procesos de enseñanza-aprendizaje. Para dar respuesta a este objetivo, a continuación se mencionan los hallazgos por cada categoría analizada.
Dentro de la categoría de apropiación tecnológica se halló que los docentes están familiarizados con herramientas tecnológicas, y hacen uso diario de la tecnología dentro de su práctica educativa. En cuanto a la integración, hacen una buena selección de los recursos por utilizar y los integran a los temas elegidos de cada materia. Finalmente, en el indicador de reorientación se encontró que los docentes entrevistados están comprometidos con brindar una excelente calidad educativa y son conscientes de que los recursos educativos abiertos ayudan a la construcción de conocimiento.

En este tema, las respuestas de los participantes coinciden con lo señalado por Colás, Rodríguez y Jiménez (2005) en cuanto a que la apropiación tecnológica contempla la manera en que adopta y se incorpora el uso de la tecnología, y que esta la hace cada individuo a partir de su propia experiencia, motivación y entorno. Así, la apropiación se percibe como una herramienta cultural, adaptada por los individuos para interpretar la realidad y ser la base del aprendizaje.

En la segunda categoría analizada, ambientes de aprendizaje, se encontró que los docentes tienen una idea de qué es un ambiente de aprendizaje, pero no conocen su concepto preciso; de la misma manera ocurrió con sus componentes, los maestros dieron sus apreciaciones pero difieren con la información recabada de los autores nombrados en el marco teórico, por ejemplo Duarte (2003), en cuanto a la concepción teórica de qué es un ambiente de aprendizaje.

Finalmente, en el análisis de la última categoría, recursos educativos abiertos, se halló que los docentes usan a la perfección dichos recursos, aun sin saber cuál era el nombre que se le daba a estos; de la misma forma, reconocen cuáles son sus características, entre las mencionadas se encuentran: fácil acceso, son gratuitos, se obtiene información de cualquier tipo, entre otras; en el uso se percibió que los REA son utilizados dentro de las clases como soporte a los temas que se enseñan y ayudan a evaluar los contenidos. 
Las respuestas de los participantes coinciden con lo expuesto por Ramírez y Burgos (2012), existe libertad en su uso, sin importar el propósito que se les dé, libre acceso, manipulación y modificación del recurso, libre distribución de la información a la cual se accede o una versión modificada de la misma.

El método de estudio de caso implementado en esta investigación, a través de las observaciones y las entrevistas, permitió un acercamiento al fenómeno en estudio para descubrir, interpretar y comprender la perspectiva de los participantes. Esta contribuyó a identificar respuestas a preguntas adicionales que emergieron en la investigación.

1) ¿Qué factores deben considerar los profesores al integrar REA en los ambientes de aprendizaje con tecnología? A partir de la información recabada los docentes consideran que los factores para tener en cuenta son: la enseñanza en cuanto a la utilización de los recursos tecnológicos, la toma de conciencia de que estos recursos no sirven solamente para diversión, sino para aprender e investigar, y, por último, que se tenga el acceso directo a dicha tecnología. 2) ¿Cuáles son las dificultades que presentan los docentes de educación básica al integrar los REA en los cursos desde la selección, integración y aplicación? Las dificultades que enfrentaron los docentes son que los recursos están en continua actualización, lo que dificulta su búsqueda; en cuanto a la integración la dificultad es el buen criterio que se debe tener para utilizar este tipo de recursos, justamente debido a que hay una variada disponibilidad de material; y por último, desde la aplicación, son dificultades de tipo técnico, en cuanto a la conectividad a la red, si el sistema de red es demasiado lento o está sobrecargado, etc.

LoS REA son un recurso educativo que tiene el potencial de impulsar el proceso de enseñanzaaprendizaje en distintos entornos y modalidades (Mortera, 2011), en la sociedad del conocimiento, ya que permiten compartir información de calidad (Rivera, López y Ramírez, 2011). Su inclusión en las actividades de enseñanza, como se encontró en este estudio de caso, genera en los docentes interés, ya que se vio a maestros activos, cuestionadores, involucrados con su proceso educativo, por lo que esperamos que esta investigación contribuya a la aceptación de la incorporación de esta poderosa herramienta del conocimiento en el aula (Atkins, Brown y Hammomd, 2007).

\section{Referencias}

Atkins, D., Brown J.y Hammomd, A. (2007). A review of the Open Educational Resources (OER) movement: Achievements, challenges, and new opportunities. Recuperado el 23 de julio de 2014 de http://goo.gl/yxklEi

Barón, L. F. y Gómez, R. (2012). De la infraestructura a la apropiación social: panorama sobre las políticas de las tecnologías de información y comunicación (TIC) en Colombia. Signo y pensamiento, 31 (61), 38-55.

Briceño, E. T. (2009). El uso del error en los ambientes de aprendizaje: una visión transdisciplinaria. Revista de Teoría y Didáctica de las Ciencias Sociales, 14, 9-28.

Celaya, R., Lozano, F.y Ramírez, M. S. (2010). Apropiación tecnológica en profesores que incorporan recursos educativos abiertos en educación media superior. Revista Mexicana de Investigación Educativa, 15 (45), 487-513. Recuperado el 23 de julio de 2014 de http://goo.gl/Z2oKgB 
ISSN $0123-1294$ | Educ.Educ. Vol. 19. No. 1 | Enero-Abril de 2016 | pp. 105-117.

Universidad de La Sabana | Facultad de Educación

Cifuentes, R. M. (2011). Diseño de proyectos de investigación cualitativa. Buenos Aires: Centro de Publicaciones Educativas y Material Didáctico.

Colás, P., Rodríguez, M. y Jiménez, R. (2005). Evaluación de e-learning. Indicadores de calidad desde el enfoque sociocultural. Teoría de la Educación y Cultura en la Sociedad de la Información, 6 (2), 3.

D’Antoni, S. (2007). Open Educational Resources and Open Content for Higher Education. Revista de Universidad y Sociedad del Conocimiento (RUSC), 4 (1), 1-7.

Duarte, J. (2003). Ambientes de aprendizaje: una aproximación conceptual. Estudios Pedagógicos, 29, 97-113.

Gómez-Zermeño, M. G. (2012). Bibliotecas digitales: modelo para el diagnóstico de recursos bibliográficos en formato electrónico disponibles para la educación básica. Monterrey: Tecnológico de Monterrey.

Gutiérrez, E. y Quiroz, R. (2007). Usos y formas de apropiación del video en una secundaria incorporada al proyecto Sec. XXI. Revista Mexicana de Investigación Educativa, 12 (32), 337-358.

Hooper, S. y Rieber, L. P. (1995). Teaching with technology. En Ornstein, A. C. (ed.). Teaching: Theory into practice (pp.154-170). Needham Heights, MA: Allyn and Bacon.

Laffey, J. (2004). Appropriation, Mastery and Resistance to Technology in Early Childhood Preservice Teacher Education. Journal of Research on Technology in Education, 36 (4), 361-382.

Lizama Mendoza, J. (2010). Phreakers y Hackers: la apropiación social de la tecnología como una nueva matriz cultural. II Congreso del Observatorio para la Cibersociedad. Recuperado el 27 de julio de 2014 de http://goo.gl/prfAeV

Mayan, M. (2001). Una introducción a los métodos cualitativos: módulo de entrenamiento para estudiantes y profesionales (eBook). Alberta: Qual Institute Press.

Ministerio de Educación Nacional (2008). Ser competentes en tecnología: ¡Una necesidad para el desarrollo! Bogotá: Espantapájaros Taller.

Montes, J. A. y Ochoa, S. (2006). The appropriation of information and communication technologies in university courses. Revista Acta Colombiana de Psicología, 9 (2), 87-100.

Mortera, F. (2011). Implementación de recursos educativos abiertos (REA) a través del portal TEMOA (Knoledge Hub) del Tecnológico de Monterrey, México. Revista formación universitaria, 3 (5), 9-20.

Mulder, F. (2007). The advancement of lifelong learning through open educational resources in an open and flexible (self) learning context. Recuperado el 27 de julio de 2014 de http://goo.gl/pE1eFr

Ramírez, M. S. (2012). Modelos y estrategias de enseñanza para ambientes innovadores. Recuperado el 23 de agosto de 2014 de http://goo.gl/LuiKKj

Ramírez, M. S. y Burgos, J. V. (coords.) (2011). Transformando ambientes de aprendizaje en educación básica con recursos educativos abiertos. México: Lulú Editorial Digital. 
Ramírez, M. S. y Burgos, J. V. (2012). Movimiento educativo abierto: acceso, colaboración y movilización de recursos educativos abiertos. México: Lulú Editorial Digital.

Ramírez, M. S. y Burgos, J. V. (coords.) (2012). Recursos educativos abiertos y móviles para la formación de investigadores: investigaciones y experiencias prácticas. México: Lulú Editorial Digital.

Rivera, R., López, A. y Ramírez, M. S. (2011). Estrategias de comunicación para el descubrimiento y uso de recursos educativos abiertos. REICE. Revista iberoamericana sobre calidad, eficacia y cambio en educación, 9 (4), 141-157.

Stake, R. (2007). Investigación con estudios de casos. Madrid: Morata.

Valenzuela, J. y Flores, M. (2012). Fundamentos de investigación educativa, vols. 2 y 3. México: Editorial Digital Tecnológico de Monterrey.

Yin, R. K. (2006). Case Study Methods. En Green, J. L., Camilli, G., Elmore, P. B. (eds.). Handbook of complementary methods in education research (pp. 111-122). Estados Unidos: Routledge. 
